\title{
Comparative transcriptome analysis of two Gerbera hybrida cultivars revealed cold tolerance mechanism
}

\author{
Shenchong $\mathrm{Li}^{1,2,3}$, Qinli Shan ${ }^{2,3}$, Rongpei $\mathrm{Yu}^{2,3}$, Abu Nasar Siddique ${ }^{4},{\mathrm{Xia} \mathrm{Li}^{2,3}, \text { Chunmei Yang }}^{2,3}$, \\ Jiwei Ruan ${ }^{2,3}$, Mingyang $\mathrm{Li}^{1 *}$ \\ ${ }^{1}$ College of Horticulture and Landscape Architecture, Southwest University, Chongqing 400715, China \\ ${ }^{2}$ Flower Research Institute, Yunnan Academy of Agricultural Sciences, Kunming 650205, China \\ ${ }^{3}$ National Engineering Research Center for Ornamental Horticulture, Kunming 650205, China \\ ${ }^{4}$ Kunming Institute of Botany, Chinese Academy of Sciences, Kunming 650201, China
}

\section{*Corresponding author: limy@swu.edu.cn}

\begin{abstract}
Temperature is one of central factors that shape the distribution and growth pattern of Gerbera hybrida. The cultivars of this species have vast variation in the morphology and stress adaptation. In the current study, comparative transcriptome analysis of two haploid cultivars with discriminative phenotype, i.e., Gerbera hybrida Hongjixing and Gerbera hybrida Qiuri, was used to uncover differentially expressed functional unigenes using an Illumina Hiseq2000 sequencing platform. Through high-throughput cDNA sequencing (RNA-seq), we generated 99160 UniGenes from $19.09 \mathrm{G}$ primary reads with an average length of $978 \mathrm{bp}$ and an N50 equaling 1620. It was filtered by Trimmomatic and assembled by Trinity. A total of 58127 transcripts were functional annotated by mapping to five protein databases by BLASTX, with topmost differently expressed unigenes (DEGs) directed to transcriptional regulation, secondary metabolism and stress response, etc. Cold treatment $\left(-2^{\circ} \mathrm{C}\right)$ of haploid seedlings in intelligent cooled incubator had adverse effects on both cultivars, which was more serious in Qiuri which was exhibited by the lower chlorophyll fluorescence after cold treatment, compared to Hongjixing. This indicates the better cold tolerance of Hongjixing. The qRT-PCR result indicated that besides common pathways, novel pathways like latex biosynthesis potentially contributes to the better cold tolerance of Hongjixing. In view of the ubiquity of cold temperature, this study provided new insights into the mechanism dissection and guidance for cold stress condition.
\end{abstract}

Keywords: Gerbera; cold stress; transcriptome; interspecies.

Abbreviations: RNA-seq_high-throughput cDNA sequencing, DEGs_differently expressed unigenes, qRT-PCR_quantitative reverse transcription-PCR.

\section{Introduction}

Gerbera hybrida, a well-known herb of family Asteraceae is famous for its medicinal as well as ornamental values (Krips et al., 2001). This herb has a rich biodiversity with specialized structures such as elaborate inflorescence, fused petals and aborted margins (Laitinen et al., 2005; Teeri et al., 2006) and strong adaptability to the environment (Bu et al., 2015). It is ranked in the top five global cut and pot flower market, together with rose, carnation, chrysanthemum and tulip (Gong and Deng, 2010).

Gerbera hybrida have been used in flower organ differentiation (Laitinen et al., 2005; Meng and Wang, 2004; Zhang et al., 2017), phytohormone regulation (Huang et al., 2017; Li et al., 2015), secondary metabolites of flavor (Deng et al., 2014) and tissue culture (Murashige et al., 1974). However, it has gained little attention with relation to stress tolerance, which could aid in identifying key functional genes and optimizing yield outputs in different environments. Gerbera hybrida is mainly planted in greenhouses. A number of modern urbanization attempts have been made to cultivate this plant in the outfield but it remained a big challenge due to low temperature during winter nights. Our field experience found that different cultivars of Gerbera hybrida show large variation in their biotic and/or environmental responses, i.e., some cultivars show growth retardation under low temperature, while others could keep flowering even below $0^{\circ} \mathrm{C}$. For example, Gerbera hybrida Hongjixing could tolerate the extreme cold temperature of $4^{\circ} \mathrm{C}$ due to its genetic potential.

RNA-seq is a comprehensive approach to track transcriptome profiling (Wang et al., 2009), which has been widely used to reveal temporal-spatial differences of gene expression (Wang et al., 2011; Wigge et al., 2005). Transcriptome analysis of the inflorescence of Gerbera hybrid uncovered novel regulators of flower type (Laitinen et al., 2005). Gene sets abundantly expressed during petal organogenesis and EST-SSR markers were also revealed by transcriptional analysis (Gong and Deng, 2010; Laitinen et al., 2007). 
However, no comparison has been made between intraspecies so far.

The natural breeding of Gerbera hybrida is timeconsuming and highly heterozygous because of its long life cycle and cross-pollination (Nagaraju et al., 1998). But haploid breeding with unfertilized pollen is an ideal material for commercial cultivation in Gerbera. In this study, two domestically cultivated Gerbera cultivars from Yunnan province of China, i.e., Gerbera hybrida Hongjixing and Gerbera hybrida Qiuri, with different cold tolerance, were compared by transcriptome analysis to uncover the coldtolerant mechanism of both cultivars. This study would provide useful guidance for future farming application practices.

\section{Results}

\section{RNA sequencing using Illumina platform and assembly of unigenes}

A total of $19.09 \mathrm{~GB}$ raw reads were generated through pairend sequencing on Illumina Hiseq2000 platform, with Q20 percentage value over $97 \%$ and moderate GC content for Gerbera hybrida Hongjixing and Gerbera hybrida Qiuri, which indicate the reliability of the quality of raw sequencing data (Table 1). After processing the raw data by Trimmomatic, clean reads were assembled de novo into 138,363 contigs and 92,606 unigenes for Hongixing, 146,762 contigs and 99,813 unigenes for Qiuri. Nucleotide length is an integrated criterion to assess the sequencing and assembly success. The average length of contig count $398 \mathrm{bp}$ with its N50 equaling $810 \mathrm{bp}$ and that of unigene reached 791 bp with its N50 equaling 1505 bp for Hongjixing, while for Qiuri it was $781 \mathrm{bp}$ and $1528 \mathrm{bp}$ as regarding to contig $\mathrm{N} 50$ and unigene N50. Size distribution of all unigenes showed that $34.64 \%$ of total unigenes had sequence length larger than $1000 \mathrm{bp}$ (Fig.S1).

\section{Functional annotation and classification of unigenes}

Function of each unigene was annotated after getting highest sequence similarity by doing BLAST to protein databases. A total of 58127 unigenes could be successfully annotated (Table 2). $\mathrm{Nr}$ database gave the highest percentage of annotation number. Further attention was paid to the quality of these annotation results. As shown in Fig.1A, all annotated unigene had e-value less than 10-5, which is a vendible threshold for sequence blast. E-value distributions indicated that $52.1 \%$ of the $\mathrm{Nr}$ annotated unigene had high similarity (E-value $<10-60)$. The similarity distribution proved that only $9.2 \%$ of annotated unigene had similarities less than $40 \%$, and $61.9 \%$ had similarities larger than $60 \%$ (Fig. 1B). Regarding the species distribution of annotated unigene, Vitis vinifera accounted for the largest proportion followed by Lycopersicon esculentum and Amygdalus persica which occupied approximately $2 / 3$ of the species number (Fig. 1C).

GO ontology is an international standard system for gene function classification, which describes the property of gene and its product with a suit of dynamic controlled vocabulary (Ashburner et al., 2000). Based on previous annotated results, we attached information to $\mathrm{GO}$ analysis module in Blast2GO software (https://www.blast2go.com/).
Compartmentalized Go term at the macro levels includes biological process, cellular component and molecular function. From the point of category view, biological process own the largest mean percentage in annotated unigenes, while cellular process, metabolic process, cell, cell part and organelle ranked the top 5 in the sublevel classification (Fig.2).

\section{Differential expressed gene (DEG) analysis}

Among the assemble outputs, 92960 unigenes were mapped to Qiuri, and 91841 unigenes on Hongjixing, respectively, with $87.1 \%$ of the unigenes coexisted in both cultivars (Fig.3A). In order to get insight into the transcriptional pattern of identified sequence, unigene expression levels were characterized by using the reads per kilobase of exon model per million mapped reads (FPKM) algorithm. The FPKM ratio of Gerbera hybrida Hongjixing versus Gerbera hybrida Qiuri was log10 plotted in Fig.3B. Taking two-fold ratio as significant criterion, we found 19100 significant upregulated and 13931 significant downregulated unigenes in Gerbera hybrida Hongjixing.

To unveil the functional significance of transcriptional level, we picked top 30 upregulated unigenes of Gerbera hybrida Hongjixing for in-depth exploration (Fig.3C). 20 out of the top 30 conspicuous upregulated unigenes were annotated by $\mathrm{Nr}$ database successfully. The gi number of the 20 annotated unigene was converted to Gene ID for online KEGG analysis (https://biodbnetabcc.ncifcrf.gov/db/db2db.php). The KEGG results showed that DEGs mainly participated in carbon metabolism (Unigene45349_All), nucleotide metabolism (Unigene 46167_All, Unigene49221_All), secondary metabolism (Unigene48068_All, Unigene49105_All), transcription regulation (Unigene44870_All, Unigene45911_All, Unigene 49221_All) and defense related response (Unigene49372_All) (Table 3). Essentially, all these unigenes could relate to multiple abiotic stress responses.

\section{Cold tolerance assay}

As cold temperature is a pivotal factor that affects the distribution of Gerbera hybrida (Broholm et al., 2008), we treated haploid Gerbera hybrida Hongjixing and Gerbera hybrida Qiuri seedlings under $-2{ }^{\circ} \mathrm{C}$ for $2 \mathrm{~h}$ in a controlled chamber, with the programme documented in (Zhang et al., 2014). Fv/Fm is a reliable indicator to reflect the photosynthetic efficiency of PSII, which is widely used in stress tolerance assays ( $\mathrm{Li}$ et al., 2006; Zhao et al., 2005; Zhou et al., 2016). Before cold treatment, both Hongjixing and Qiuri showed normal growth, with high photosynthetic potential indicated by blue color (Fig.4A) but retarded growth was observed in both Hongjixing and Qiuri. However, Hongjixing survived better after cold stress, as reflected by high photosynthetic potential (Fig.4B). Leaf margins of Qiuri almost died and only the main stem remained alive.

\section{Expression profile of candidate DEGs in response to cold treatment}

To validate the crucial role of DEGs in response to low temperature, we carried out qRT-PCR analysis. After cold treatment, the qRT-PCR results showed that 12 out of 20 
Table 1. Quality index of transcriptome sequencing and de novo assembly.

\begin{tabular}{lllll}
\hline & & Gerbera_hybrida Hongjixing Gerbera_hybrida QiuriAll \\
\hline \multirow{3}{*}{ Reads } & Total reads & $111,868,608$ & $113,041,480$ & - \\
& Clean reads & $105,625,976$ & $106,538,440$ & - \\
& Total clean nucleotides (nt) & $9,506,337,840$ & $9,588,459,600$ & - \\
\hline Paired-end percentage(\%) & $83.81 \%$ & $76.98 \%$ & - \\
Q20 percentage (\%) & 97.82 & 97.97 & - \\
N percentage (\%) & 0 & 0 & - \\
GC percentage (\%) & 44.83 & 44.55 & - \\
Contig & 138,363 & 146,762 & - \\
\hline Total number & 398 & 396 & - \\
Mean length & 810 & 781 & - \\
N50 & 92,606 & 99,813 & 99,160 \\
\hline Unigene Total number & 791 & 802 & 978 \\
Mean length & 1505 & 1528 & 1620 \\
\hline
\end{tabular}

(A) E-value Distribution

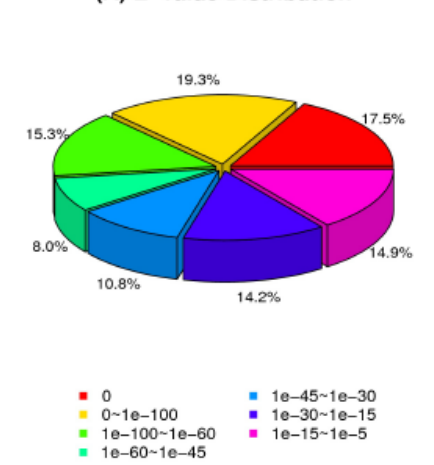

(C) Species Distribution

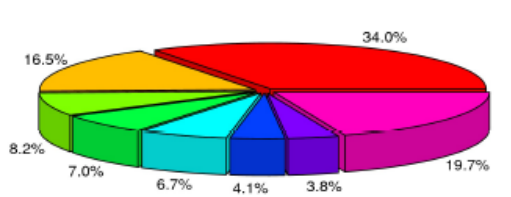

(B) Similarity Distribution

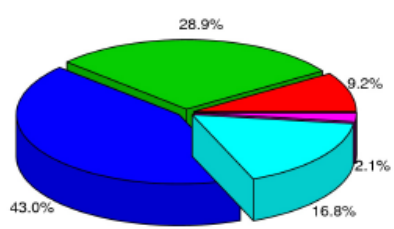

$\begin{array}{ll}-17 \% \sim 40 \% & =80 \% \sim 95 \% \\ 40 \% \sim 60 \% & =95 \% \sim 100 \%\end{array}$

Fig 1. Annotation of unigenes according to $\mathrm{Nr}$ database. (A) E-value distribution according to Blast comparsion annotion with $\mathrm{Nr}$ database. All the annotated unigenes have a significant E-value; (B) Similarity distribution according to Blast comparsion annotion with $\mathrm{Nr}$ database, around two thirds of unigenes have a similarity higher than 60\%; (C) Unigenes in our data have a best hit genes in others species according to Blast comparsion annotion with $\mathrm{Nr}$ database, Vitis vinifera genome offers the most annotation.

annotated DEGs showed significant expression variation between Gerbera hybrida Hongjixing and Gerbera hybrida Qiuri. Among them, Unigene49221, Unigene49372, Unigene50734, Unigene44870, Unigene45349, Unigene45911, Unigene46132, Unigene48047, Unigene48068, Unigene48783 and Unigene49105 of Gerbera hybrida Hongjixing had significant upregulation as compared to Gerbera hybrida Qiuri (Fig.4), which clearly reflects their potential function in cold tolerance. However, these unregulated DEGs were mainly involved in nucleotide metabolism, transcriptional regulation, secondary metabolite biosynthesis and stress response.

\section{Discussion}

Cold tolerance facilitates the planting feasibility and international influence of Gerbera hybrida. In our previous breeding practices, Hongjixing was selected as cultivar candidate for abundant flowers yield and strong stress resistance (Yu, 2014). In Yunnan, cold stress became a major factor preventing the Gerbera hybrida for outfield planting due to the temperature difference between day and night during winter. As Hongjixing and Qiuri showed growth tendency in winter, we carried out transcriptome analysis between these cultivars. For Gerbera hybrida, we assembled de novo transcriptome sequences in Trinity which was based on raw data, because of the non-availability of referenced genome information for Gerbera hybrida. In total, 99,160 unigenes were assembled from approximately 105 thousand clean reads, equivalent to 92 million contigs (Table 1 ). The numbers of unigenes and contigs are almost two times than the ray florets transcriptome analysis of Kuang et al. (2013), which may be ascribed to the tissue-specific expression or sequencing depth. Within these unigenes, $34 \%$ were similar to vitis vinifera (vitaceae) and $16.5 \%$ were homologous to 
Table 2. Statistics of the TOP30 unregulated DEGs of Gerbera hybrida Hongjixing.

\begin{tabular}{|c|c|c|c|}
\hline gene-ID & $\mathrm{Nr}-\mathrm{ID}$ & Nr-annotation & Ratio \\
\hline Unigene45349 & gi|255557953|ref|XP_002520005.1| & $\begin{array}{l}\text { Thermosensitive gluconokinase, putative [Ricinus communis] } \\
\text { >gi|223540769|gb|EEF42329.1| Thermosensitive gluconokinase, } \\
\text { putative [Ricinus communis] }\end{array}$ & 10.36 \\
\hline Unigene46934 & -- & -- & 10.07 \\
\hline Unigene50753 & gi|312599845|gb|ADQ91848.1| & $\begin{array}{l}\text { late embryogenesis abundant protein group } 9 \text { protein [Arachis } \\
\text { hypogaea] }\end{array}$ & 9.23 \\
\hline Unigene45440 & gi|225461754|ref|XP_002285533.1| & $\begin{array}{l}\text { PREDICTED: uncharacterized protein LOC100252018 [Vitis vinifera] } \\
\text { >gi|302142861|emb|CBI20156.3| unnamed protein product [Vitis } \\
\text { vinifera] }\end{array}$ & 8.99 \\
\hline Unigene49372 & gi|37778025|gb|AAR02572.1| & Resistance protein candidate RGC2 [Lactuca sativa] & 8.78 \\
\hline Unigene46846 & -- & -- & 8.73 \\
\hline Unigene46617 & -- & -- & 8.72 \\
\hline Unigene44870 & gi|32698524|gb|AAP86602.1| & Eukaryotic translation initiation factor 4E [Lactuca sativa] & 8.67 \\
\hline Unigene50192 & gi|225425076|ref|XP_002271486.1| & $\begin{array}{l}\text { PREDICTED: ankyrin repeat-containing protein At3g12360-like [Vitis } \\
\text { vinifera] }\end{array}$ & 8.63 \\
\hline Unigene50625 & -- & -- & 8.61 \\
\hline Unigene45932 & -- & -- & 8.60 \\
\hline Unigene50761 & -- & -- & 8.59 \\
\hline Unigene48073 & -- & -- & 8.22 \\
\hline Unigene47007 & gi|356516321|ref|XP_003526844.1| & PREDICTED: uncharacterized protein LOC100802446 [Glycine max] & 8.20 \\
\hline Unigene50734 & gi|462405507|gb|EMJ10971.1| & Hypothetical protein PRUPE_ppa013483mg [Prunus persica] & 8.18 \\
\hline Unigene50730 & gi|75223179|sp|080362.1|RK10_TOBAC & $\begin{array}{l}\text { RecName: Full=50S ribosomal protein L10, chloroplastic; AltName: } \\
\text { Full=CL10; Flags: Precursor >gi|3298441|dbj|BAA31511.1| chloroplast } \\
\text { ribosomal protein L10 [Nicotiana tabacum] }\end{array}$ & 8.13 \\
\hline Unigene46167 & gi|462396571|gb|EMJ02370.1| & Hypothetical protein PRUPE_ppa003624mg [Prunus persica] & 8.12 \\
\hline Unigene49221 & gi|460377394|ref|XP_004234478.1| & $\begin{array}{l}\text { PREDICTED: DNA-directed RNA polymerases I, II, and III subunit rpabc3- } \\
\text { like isoform } 1 \text { [Solanum lycopersicum] } \\
\text { >gi|460377396|ref|XP_004234479.1| PREDICTED: DNA-directed RNA } \\
\text { polymerases I, II, and III subunit rpabc3-like isoform } 2 \text { [Solanum } \\
\text { lycopersicum] }\end{array}$ & 8.10 \\
\hline Unigene48714 & gi|462405306|gb|EMJ10770.1| & Hypothetical protein PRUPE_ppa011099mg [Prunus persica] & 8.03 \\
\hline Unigene28 & -- & -- & 8.01 \\
\hline Unigene48783 & gi|147798869|emb|CAN61630.1| & Hypothetical protein VITISV_003191 [Vitis vinifera] & 7.98 \\
\hline
\end{tabular}




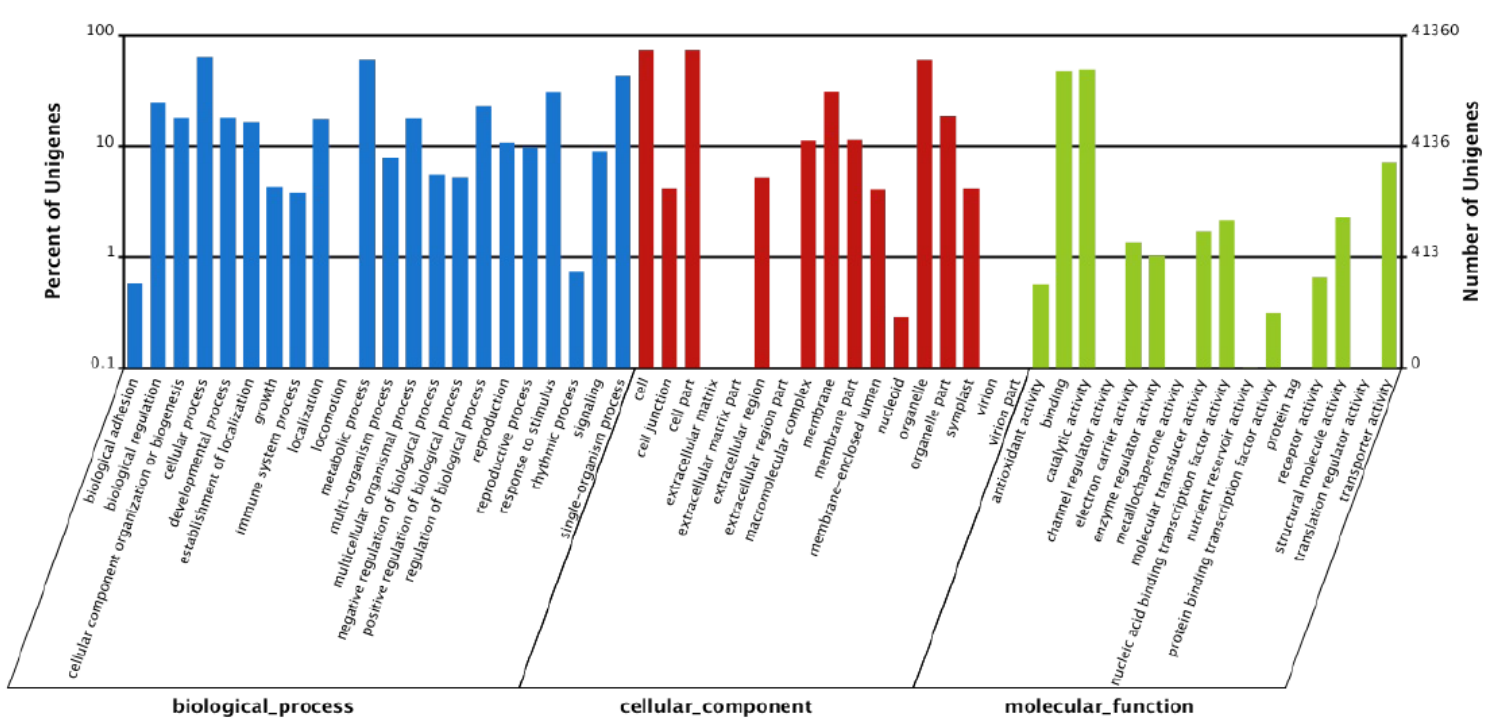

Fig 2. GO functional classification of annotated unigenes according to the transcriptome data.

A

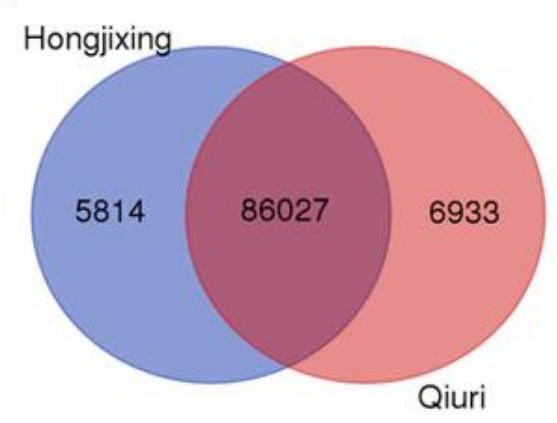

B

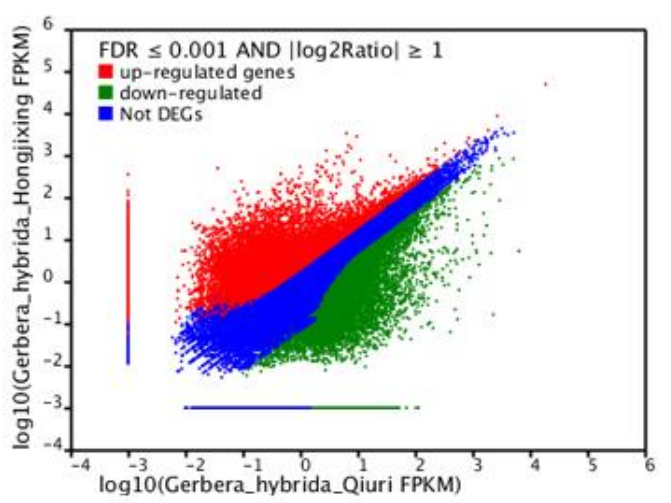

C

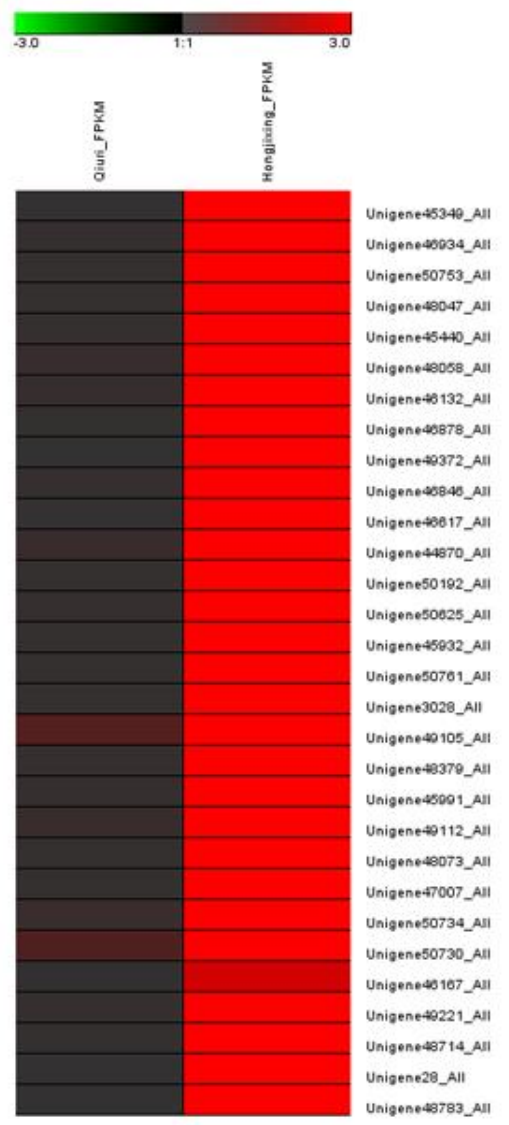

Fig 3. (A) The Venn diagram shows the number of transcripts overlap between Gerbera hybrida Hongjixing and Gerbera hybrida Qiuri. (B) Log conversion of FPKM in both species. Red dots denote significant upregulated unigene in Gerbera hybrida Hongjixing, green dots mean significant downregulated unigene in Gerbera hybrida Qiuri, blue dots marked insignificant unigenes. (C) The heatmap for the top 30 upregulated DEGs of Gerbera hybrida Hongjixing. 

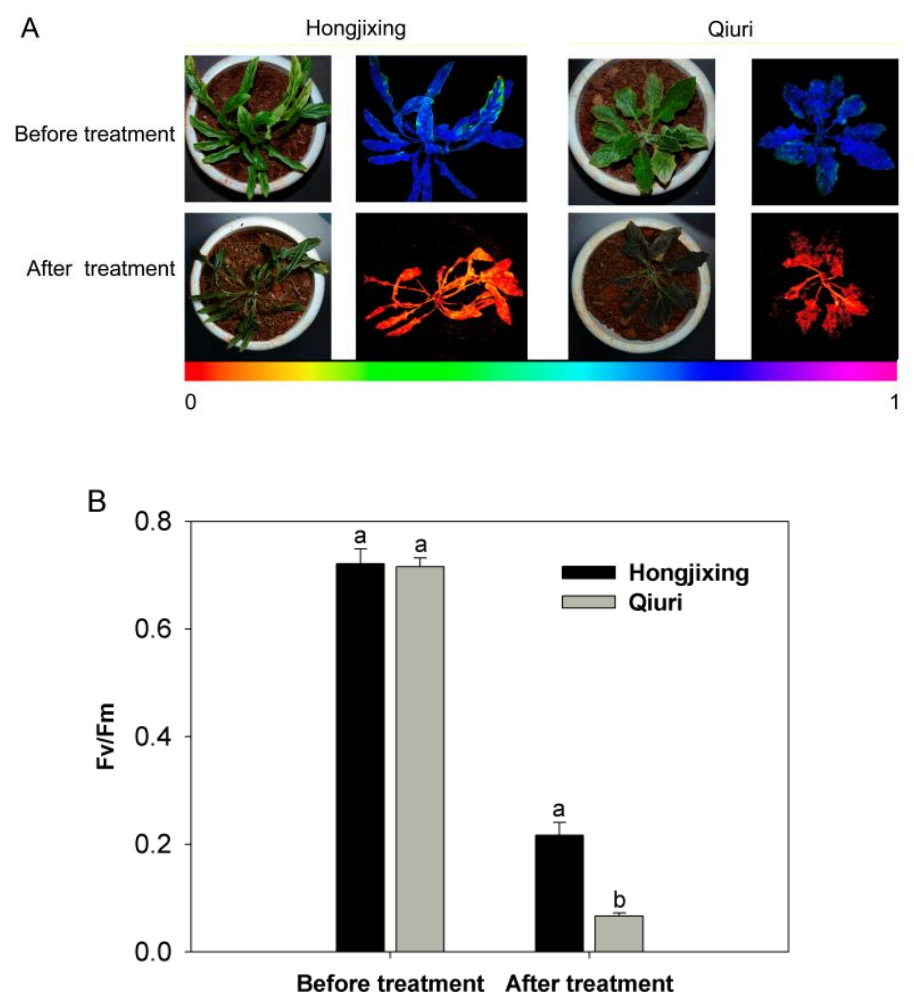

Fig 4. (A) Effect of cold treatment on the phenotype of Hongjixing and Qiuri. The second and fourth col-group pictures are taken by chlorophyll fluorescence imaging. The pseudo-color code on the bar ranged from 0 (red) to 1 (purple) indicating the maximum quantum yield of PSII. (B) Quantified statistic of Fv/Fm value before and after cold treatment. Each treatment replicated 3 times, with the bar on the column stand for SD.

lycopersicon esculentum (Solanaceae), implying potential relative phylogenetic relationship of these two species with Gerbera hybrida, presumably supporting the taxonomy of Basavaraju et al. (2009) and Sarkar (1993).

Comparative transcriptomics is widely used in identifying key points and functional revealing (Koenig et al., 2013; Rossouw et al., 2008), even within species (Davidson et al., 2012). Novel floral fragrance-related genes between two rose plants were revealed by Lewinsohn (2012) through genomic expression data analysis. In our study, more than 13 thousand unigenes expressed differently $(\log 2$ ratio $>1)$ between Hongjixing and Qiuri, with 2427 unigenes only expressed in Qiuri (data not show). Considering the positive function of the candidate unigenes, we focused on the top 30 upregulated unigenes that co-expressed in both species. Apart from 10 unigenes with none functional annotation, it was presumed that remaining 20 annotated DEGs played crucial role in shaping adaptive strategies in Hongjixing, thus further qRT-PCR analysis was applied to validate their expression patterns in response to cold stress. The results showed that seventeen out of the twenty DEGs expressed more in Hongjiixing than in Qiuri. A hypothetical vitis protein with none functional characterization (unigene 48783) and a predicted protein that supposed to functions in RNA degradation (unigene 45911) showed contrasting expression level during cold stress response (Fig.5). Resistant protein (unigene 49372), a putative eukaryotic translation initiation factor $4 \mathrm{E}$ that is responsible for RNA transport (unigene 44870), an unknown protein (unigene 50734) and a Chalcone synthase that is involved in flavonoid biosynthesis (unigene 49105) hold expression difference at nearly hundred times. Chalcone synthase related enzyme was reported to mediate flavonoid biosynthesis, which is a kind of defense compounds responsible for stress stimulus (Dao et al., 2011; Fini et al., 2011; Richard et al., 2000; WinkelShirley, 2002; Yang et al., 2002). A gluconokinase in pentose phosphate pathway (unigene 45349) was thought to counter against oxidative stress and assist photosynthesis under stress condition (Abdeen et al., 2010; Kruger and von Schaewen, 2003; Lejay et al., 2008; Savitch et al., 2001). This is in line with the higher chlorophyll fluorescence (Fv/Fm) of Hongjixing (Fig.5). A putative unigene latex-like protein (unigene 48058) was also supposed to cause a better cold tolerance of Hongjixing, which was extracted from Calotropis procera and reported for protection against inflammation and oxidative stress (Kumar and Roy, 2007), but its biological function of engagement in cold response have never been reported before. Two unigenes (Unigene44870 and Unigene45991) involved in RNA regulation were found to be active in cold tolerance. Based on the cognition that genes with similar expression patterns usually exhibit functional correlation (Ren et al., 2014), we speculated that some of these upregulated unigenes comprised in the same cold tolerance pathway. 

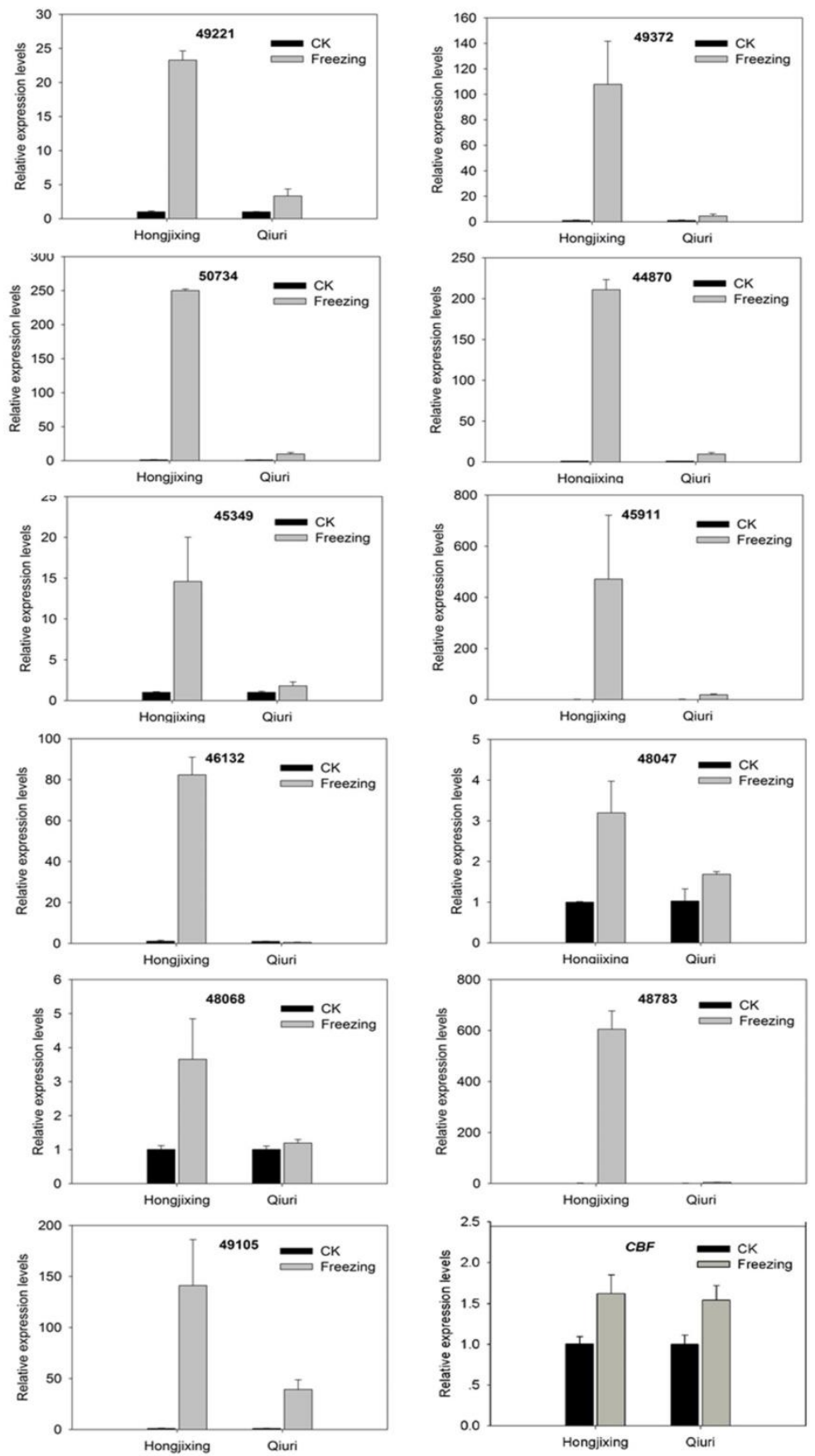

Fig 5. Relative expression levels of 12 DEGs in response to cold stress. DEGs, differently expressed unigenes. CK, control treatment with both cultivars grow under normal growth condition. Each treatment replicated 3 times, with the bar on the column stand for SD.

The ICE-CBF-COR pathway is well conserved component that contribute to cold tolerance across numerous species (Chinnusamy et al., 2007; Cook et al., 2004; Jiang et al., 2011; Thomashow et al., 2001). However, for the wellknown fatal CBF factor that is involved in cold-tolerance pathway (Chinnusamy et al., 2007; Novillo et al., 2004; Novillo et al., 2007), we did not observe significant expression difference between Hongjixing and Qiuri. Regarding cold tolerance via novel secondary metabolites (ie, latex) such as production or other unknown pathways, we speculated that Hongjixing outfit other cultivars (like
Qiuri). Nevertheless, none of these key candidates could be functionally characterized in vivo,

efficient approaches are required to identify the biologicalfunctions of these eminent genes.

\section{Materials and methods}

\section{Plant materials}

Haploid seedlings of two Gerbera hybrida cultivars were successfully regenerated by culturing the unfertilized pollen of diploid parent. Plants were grown in a phytotron with 
constant temperature of $22^{\circ} \mathrm{C}$ and photoperiod of $16 \mathrm{~h}$ light/ $8 \mathrm{~h}$ dark. After two months under the normal condition, healthy seedlings with uniform growth were sampled, frozen in liquid nitrogen and prepared to RNA-seq analysis.

\section{Cold treatments}

To test the difference between the cold-tolerance between the two Gerbera hybrida cultivars, two-month-old seedlings with uniform growth were subjected to cold treatment of $2^{\circ} \mathrm{C}$ for $2 \mathrm{~h}$ in an intelligent cooled incubator (Rumed 3301). Samples were taken before and after treatments, all treatments were replicated 3 times.

\section{RNA extraction}

Two cultivars of Gerbera, Gerbera hybrida Hongjixing and Gerbera hybrida Qiuri were used in this study. Five cultured plants of haploid Gerbera hybrida Hongjixing and Gerbera hybrida Qiuri were collected from tissue cultured sprouts. It was quick-freezed in liquid nitrogen. Total RNA of these samples was extracted using TRIZOL method (Zhou et al., 2016). The concentration and quality of RNA was evaluated by spectrophotometer NanoDrop ND-1000 at 260/230 and 260/280.

\section{cDNA library construction}

Sequencing library was constructed following the manufacturer's instruction of Illumina (San Diego, CA, USA). The mRNA with poly-A tail was enriched from total RNA by Magnetic Oligo (dT) beads and pulled down with Tris- $\mathrm{HCl}$ buffer. The eluted RNA was subjected to sonication, to break it into smaller fragments of 100-500 bp. Fragmented mRNA was then reverse transcribed into cDNA using SuperScript cDNA Synthesis kit (Invitrogen, Carlsbad, CA, USA). Double stranded cDNA fragments were purified using QiaQuick PCR extraction kit. These fragments were then fused with adapter which served as templates during PCR amplification. CDNA library was constructed with these PCR products and loaded into the flow cells.

\section{Sequencing, de novo assembly and annotation}

The cDNA library was end-paired sequenced from the adapter-fused fragments on Illumina HiSeqTM 2000 according to the manufacturer's instructions. Raw reads were first filtered for the quality control of the raw data, using the default parameters set in the Illumina, i.e., unknown nucleotides ( $N>5 \%)$. The adapters, fragment with size less than $10 \mathrm{bp}$ and low-quality reads were discarded, then subjected to reads trimming in Trimmomatic to get clean reads. Clean reads were assembled de novo by Trinity. The Trinity make up of three modules and concatenate clean reads automatically, to form Contigs and unigenes (Grabherr et al., 2011). These reads were mapped again to the contigs, until paired-end reads do not connect with any contigs at either end; these fully extended contigs were called unigenes. The unigenes were further subjected to redundancy removal, which is delimited by $94 \%$ identity within fragments larger than $100 \mathrm{bp}$.

Non-redundant unigenes were submitted to $\mathrm{Nr}$, SwissProt, KEGG or COG databases for homolog search and annotation comparison based on BLAST algorithm, with threshold e-value $\leq 10-5$. Meanwhile, unigenes were maped to nucleotide database Nt used to consolidate the BLASTX results. When a unigene was unmapped to any aforementioned databases, ESTScan were used to predict the coding sequences. Gene ontology (GO) annotation and functional classification was carried out in Blast2GO and WEGO software.

\section{Differential expressed candidate unigene screen and its pathway analysis}

Expression pattern of unigenes were compared between Gerbera hybrida Hongjixing and Gerbera hybrida Qiuri based on FPKM ratio. Variation larger than 2 times and FDR $\leq 0.001$ was considered as criterion to screen differential expressed unigene. More stringent standards were used to refrain false and ambiguous annotations by rechecking it online by BLASTX in $\mathrm{Nr}$ database. For better understanding the biological property of these differential unigene, we investigated corresponding metabolic pathway that were involved in by the KEGG analysis.

Quantitative reverse transcription polymerase chain reaction ( $q R T-P C R$ ) analysis of screened unigene

Better cold tolerance of Gerbera hybrida Hongjixing in winter motivated us to explore its underlying mechanism in response to cold/freezing treatment. Expression profiles of the top 30 differentially expressed unigene were investigated both in Gerbera hybrida Hongjixing and Gerbera hybrida Qiuri, then both cultivars (one-month-old haploid sprouts) were exposed to $-2^{\circ} \mathrm{C}$ for two hours and then it was recovered at room temperature for one week. Samples were collected at indicated time and frozen in liquid nitrogen. The total RNA was isolated by TRIZOL, $2 \mu \mathrm{g}$ total RNA was reverse-transcribed to CDNA as mentioned above. This product was used as template in real time PCR. Primers of these unigene were located in the un-conserved region for specificity and listed in Table S1.

\section{Statistical analysis}

This study use fragments per kilobase of exon model per million mapped (FPKM) to normalize the expression levels of transcripts. The identification of differently expressed unigenes (DEGs) was filtered by the ratio of log-transformed FPKM and false discovery rate (FDR). For testing the effect of cold stress on the expression of candidate DEGs, qRT-PCR results were compared between before and after cold treatment with one way ANOVA.

\section{Acknowledgments}

This work was financially supported by The National Key Technology R\&D Program (No. 2015BAD10B00). We thank Dr. Abu Nasar Siddique for the critical reading of this manuscript.

\section{References}

Abdeen A, Schnell J \& Miki B (2010) Transcriptome analysis reveals absence of unintended effects in drought-tolerant 
transgenic plants overexpressing the transcription factor ABF3. BMC genomics. 11(1):1-21.

Ashburner M, Ball CA, Blake JA, Botstein D, Butler $\mathrm{H}$, Cherry JM, Davis AP, Dolinski K, Dwight SS, Eppig JT, Harris MA, Hill DP, Issel-Tarver L, Kasarskis A, Lewis S, Matese JC, Richardson JE, Ringwald M, Rubin GM \& Sherlock G (2000) Gene ontology: tool for the unification of biology. Nat Genet. 25(1):25-29.

Basavaraju R, Raj JV \& Bhiravamurthy P (2009) Medicinal plant resources of Puttaparthi Mandal: taxonomic overview and need for conservation. Ethnobotanical Leaflets. 2009(11):6.

Broholm SK, Tähtiharju S, Laitinen RA, Albert VA, Teeri TH \& Elomaa P (2008) A TCP domain transcription factor controls flower type specification along the radial axis of the Gerbera (Asteraceae) inflorescence. Proc Natl Acad Sci USA. 105(26):9117-9122.

Bu X, Gao Y, Fan M \& Zhang Q Application of transcriptome sequencing in molecular breeding in Asteraceae plants. In, 2015. International Society for Horticultural Science (ISHS), Leuven, Belgium, p 59-63.

Chinnusamy V, Zhu J \& Zhu J-K (2007) Cold stress regulation of gene expression in plants. Trends Plant Sci. 12(10):444451.

Cook D, Fowler S, Fiehn O \& Thomashow MF (2004) A prominent role for the CBF cold response pathway in configuring the low-temperature metabolome of Arabidopsis. Proc Natl Acad Sci USA. 101(42):15243-15248.

Dao TTH, Linthorst HJM \& Verpoorte R (2011) Chalcone synthase and its functions in plant resistance. Phytochem Rev. 10(3):397.

Davidson RM, Gowda M, Moghe G, Lin H, Vaillancourt B, Shiu SH, Jiang N \& Robin Buell C (2012) Comparative transcriptomics of three Poaceae species reveals patterns of gene expression evolution. Plant J. 71(3):492-502.

Deng $X$, Bashandy $H$, Ainasoja $M$, Kontturi J, Pietiäinen $M$, Laitinen RAE, Albert VA, Valkonen JPT, Elomaa P \& Teeri TH (2014) Functional diversification of duplicated chalcone synthase genes in anthocyanin biosynthesis of Gerbera hybrida. New Phytol. 201(4):1469-1483.

Fini A, Brunetti C, Di Ferdinando $M$, Ferrini F \& Tattini $M$ (2011) Stress-induced flavonoid biosynthesis and the antioxidant machinery of plants. Plant Signal Behav. 6(5):709-711.

Gong L \& Deng Z (2010) EST-SSR markers for gerbera (Gerbera hybrida). Mol Breeding. 26(1):125-132.

Grabherr MG, Haas BJ, Yassour M, Levin JZ, Thompson DA, Amit I, Adiconis X, Fan L, Raychowdhury R, Zeng Q, Chen Z, Mauceli E, Hacohen N, Gnirke A, Rhind N, di Palma F, Birren BW, Nusbaum C, Lindblad-Toh K, Friedman N \& Regev A (2011) Trinity: reconstructing a full-length transcriptome without a genome from RNA-Seq data. Nat Biotechnol. 29(7):644-652.

Huang G, Han M, Yao W \& Wang Y (2017) Transcriptome analysis reveals the regulation of brassinosteroids on petal growth in Gerbera hybrida. PeerJ. 5:e3382.

Jiang F, Wang F, Wu Z, Li Y, Shi G, Hu J \& Hou X (2011) Components of the Arabidopsis CBF cold-response pathway are conserved in non-heading chinese cabbage. Plant Mol Biol Rep. 29(3):525-532.

Koenig D, Jiménez-Gómez JM, Kimura S, Fulop D, Chitwood DH, Headland LR, Kumar R, Covington MF, Devisetty UK, Tat AV, Tohge T, Bolger A, Schneeberger K, Ossowski S,
Lanz C, Xiong G, Taylor-Teeples M, Brady SM, Pauly M, Weigel D, Usadel B, Fernie AR, Peng J, Sinha NR \& Maloof JN (2013) Comparative transcriptomics reveals patterns of selection in domesticated and wild tomato. Proc Natl Acad Sci USA. 110(28):E2655-E2662.

Krips OE, Willems PEL, Gols R, Posthumus MA, Gort G \& Dicke M (2001) Comparison of cultivars of ornamental crop Gerbera jamesonii on production of spider miteinduced volatiles, and their attractiveness to the predator, Phytoseiulus persimilis. J Chem Ecol. 27(7):1355-1372.

Kruger NJ \& von Schaewen A (2003) The oxidative pentose phosphate pathway: structure and organisation. Curr Opin Plant Biol. 6(3):236-246.

Kuang Q, Li L, Peng J, Sun S \& Wang X (2013) Transcriptome analysis of Gerbera hybrida ray florets: putative genes associated with gibberellin metabolism and signal transduction. PLoS One. 8(3):e57715.

Kumar VL \& Roy S (2007) Calotropis procera latex extract affords protection against inflammation and oxidative stress in Freund's complete adjuvant-induced monoarthritis in rats. Mdiat Inflamm. 2007.

Laitinen RAE, Immanen J, Auvinen P, Rudd S, Alatalo E, Paulin L, Ainasoja M, Kotilainen M, Koskela S, Teeri TH \& Elomaa P (2005) Analysis of the floral transcriptome uncovers new regulators of organ determination and gene families related to flower organ differentiation in Gerbera hybrida (Asteraceae). Genome Res. 15(4):475-486.

Laitinen RAE, Pöllänen E, Teeri TH, Elomaa P \& Kotilainen M (2007) Transcriptional analysis of petal organogenesis in Gerbera hybrida. Planta. 226(2):347-360.

Lejay L, Wirth J, Pervent M, Cross JM-F, Tillard P \& Gojon A (2008) Oxidative pentose phosphate pathway-dependent sugar sensing as a mechanism for regulation of root ion transporters by photosynthesis. Plant Physiol. 146(4):2036-2053.

Lewinsohn E (2012) Rose scent: genomics approach to discovering novel floral fragrance-related genes. Plant Cell. 14(10):2325-2338.

Li L, Zhang W, Zhang L, Li N, Peng J, Wang Y, Zhong C, Yang Y, Sun S, Liang S \& Wang X (2015) Transcriptomic insights into antagonistic effects of gibberellin and abscisic acid on petal growth in Gerbera hybrida. Front Plant Sci. 6:168.

Li R-H, Guo P-G, Michael B, Stefania G \& Salvatore C (2006) Evaluation of chlorophyll content and fluorescence parameters as indicators of drought tolerance in barley. Agr Sci China. 5(10):751-757.

Meng X \& Wang X (2004) Regulation of flower development and anthocyanin accumulation in Gerbera hybrida. J Hortic Sci Biotech. 79(1):131-137.

Murashige T, Serpa M \& Jones JB (1974) Clonal multiplication of Gerbera through tissue culture. Hortic Sci. 9:175-180.

Nagaraju V, G.S.L. S \& G. LS (1998) Agrobacterium -mediated genetic transformation in Gerbera hybrida. Curr Sci India. 74(7):630-634.

Novillo F, Alonso JM, Ecker JR \& Salinas J (2004) CBF2/DREB1C is a negative regulator of CBF1/DREB1B and CBF3/DREB1A expression and plays a central role in stress tolerance in Arabidopsis. Proc Natl Acad Sci USA. 101(11):3985-3990.

Novillo F, Medina J \& Salinas J (2007) Arabidopsis CBF1 and CBF3 have a different function than $\mathrm{CBF} 2$ in cold 
acclimation and define different gene classes in the CBF regulon. Proc Natl Acad Sci USA. 104(52):21002-21007.

Ren L, Sun J, Chen S, Gao J, Dong B, Liu Y, Xia X, Wang Y, Liao Y, Teng N, Fang W, Guan Z, Chen F \& Jiang J (2014) A transcriptomic analysis of Chrysanthemum nankingense provides insights into the basis of low temperature tolerance. BMC Genomics. 15(1):844.

Richard S, Lapointe G, Rutledge RG \& Séguin A (2000) Induction of chalcone synthase expression in white spruce by wounding and jasmonate. Plant Cell Physiol. 41(8):982987.

Rossouw D, Næs T \& Bauer FF (2008) Linking gene regulation and the exo-metabolome: a comparative transcriptomics approach to identify genes that impact on the production of volatile aroma compounds in yeast. BMC genomics. 9(1):1.

Sarkar S (1993) Studies on herbaceous plants of Karbi Anglong district of Assam with reference to their taxonomy and economic utilisation. Ph.D thesis, Gauhati University

Savitch LV, Barker-Åstrom J, Ivanov AG, Hurry V, Öquist G, Huner NP \& Gardeström P (2001) Cold acclimation of Arabidopsis thaliana results in incomplete recovery of photosynthetic capacity, associated with an increased reduction of the chloroplast stroma. Planta. 214(2):295303.

Teeri TH, Kotilainen M, Uimari A, Ruokolainen S, Peng Ng Y, Malm U, Pöllänen E, Broholm S, Laitinen R, Elomaa P \& Albert VA (2006) Floral developmental genetics of Gerbera (Asteraceae). Adv Bot Res. Volume 44:323-351.

Thomashow MF, Gilmour SJ, Stockinger EJ, Jaglo-Ottosen KR \& Zarka DG (2001) Role of the Arabidopsis CBF transcriptional activators in cold acclimation. Physiol Plantarum. 112(2):171-175.
Wang D, Pan Y, Zhao X, Zhu L, Fu B \& Li Z (2011) Genomewide temporal-spatial gene expression profiling of drought responsiveness in rice. BMC Genomics. 12(1):149.

Wang Z, Gerstein M \& Snyder M (2009) RNA-Seq: a revolutionary tool for transcriptomics. Nat Rev Genet. 10(1):57-63.

Wigge PA, Kim MC, Jaeger KE, Busch W, Schmid M, Lohmann JU \& Weigel D (2005) Integration of spatial and temporal information during floral induction in Arabidopsis. Science. 309(5737):1056-1059.

Winkel-Shirley B (2002) Biosynthesis of flavonoids and effects of stress. Curr Opin Plant Biol. 5(3):218-223.

Yang J, Huang J, Gu H, Zhong Y \& Yang Z (2002) Duplication and adaptive evolution of the chalcone synthase genes of Dendranthema (Asteraceae). Mol Biol Evol. 19(10):17521759.

Yu L (2014) Outfield plant trials for Gerbera hybrida in Yunnan (in chiense). China Flow Hortic. 1(1):38.

Zhang J, Zhang X, Wang R \& Li W (2014) The plasma membrane-localised Ca2+-ATPase ACA8 plays a role in sucrose signalling involved in early seedling development in Arabidopsis. Plant Cell Rep. 33(5):755-766.

Zhang T, Zhao Y, Juntheikki I, Mouhu K, Broholm SK, Rijpkema AS, Kins L, Lan T, Albert VA, Teeri TH \& Elomaa P (2017) Dissecting functions of SEPALLATA-like MADS box genes in patterning of the pseudanthial inflorescence of Gerbera hybrida. New Phytol:1-16.

Zhao L, Deng X \& Shan L (2005) Effects of osmotic stress on chlorophyll fluorescence parameters of wheat seedling (in chinese). Chin J Appli Ecol. 16(7):1261-1264.

Zhou Y, Sun X, Yang Y, Li X, Cheng Y \& Yang Y (2016) Expression of Stipa purpurea SpCIPK26 in Arabidopsis thaliana enhances salt and drought tolerance and regulates abscisic acid signaling. In J Mol Sci. 17(6):966. 